\title{
Bimekizumab: the first bispecific biologic agent facing phase III trial program in both plaque psoriasis and psoriatic arthritis
}

\author{
Chiricozzi A and Romanelli M* \\ Dermatology Unit, Department of Clinical and Experimental Medicine, University of Pisa. Via Roma 67, 56126 PISA, Italy
}

\section{Introduction}

Psoriasis is a chronic inflammatory skin disorder that in at least $20 \%$ of cases could be associated with arthritis (PsA) [1]. Skin and joint inflammation is driven by a wide array of pathways, though multiple lines of evidence supported the pathogenic model centered on the interleukin (IL)-23/IL-17A axis [2]. Beside IL-23/IL-17 axis playing a pivotal role in psoriasis pathogenesis, other cytokines with pro-inflammatory and/or pro-proliferative activity contribute to the development of the psoriatic phenotype [2]. Among them, IL$17 \mathrm{~F}$, showing greater than $50 \%$ structural homology and overlapping biological function with IL-17A, results 30 -fold less biologically active than IL-17A [3,4]. Both IL-17A and IL-17F are increasingly expressed in psoriatic lesional skin and inflamed synovium from PsA [5-8]. Similarly, to IL-17A, IL-17F can cooperate with TNF in inducing the production of key pro-inflammatory cytokines, and thus, amplifying tissue inflammation [5-7]. The dual blockade of IL-17A and IL-17F induces a more potent suppression of inflammatory signals, compared to the selective IL-17A or IL-17F inhibition, reducing the expression of inflammation-linked genes and cytokines, as well as the disease-relevant immune cell migration, at greater extent [5-7]. These data provided the rationale for developing a bispecific humanized monoclonal IgG1 antibody, named bimekizumab, that can simultaneously neutralize both IL-17A and IL-17F.

Clinical studies testing bimekizumab for the treatment of psoriasis: The first-in-human, placebo-controlled, single-doseescalating study randomizing 13 subjects to placebo and to escalating doses of bimekizumab ( $8 \mathrm{mg}, 40 \mathrm{mg}, 160 \mathrm{mg}, 480 \mathrm{mg}$, and $640 \mathrm{mg}$ ), showed promising clinical outcomes [9]. All subjects receiving a single bimekizumab dose at the baseline were followed for 20 weeks. Clinical response was detected with higher bimekizumab doses $(160 \mathrm{mg}, 480$ $\mathrm{mg}$, and $460 \mathrm{mg}$ ), by week 2 , reaching the maximal improvement between week 4 and 6 that was maintained through 16-20 weeks. In patients treated with $480 \mathrm{mg}$, and $460 \mathrm{mg}$ bimekizumab injection, the reduction of disease severity estimated as at least $90 \%$ improvement of the baseline psoriasis index severity area (PASI) score (PASI 90 response), was achieved by $83 \%$ of patients from week $6-12$, and in $90 \%$ at week 12 . Overall, 78 adverse events occurring in all 39 participants (including placebo- and bimekizumab-treated subjects) were classified as mild or moderate, except one. The serious adverse event was not classified as treatment-related: it was observed in one $40 \mathrm{mg}$ bimekizumab-treated patient and it consisted of vomiting that required hospitalization. Subsequently, a multi-center, randomized, double-blinded, placebo-controlled, parallel-group, dose-ranging, phase IIb study (named BE ABLE trial) tested bimekizumab vs. placebo in adult patients with moderate to severe chronic plaque psoriasis [10]. The study included a 12-week treatment period followed an extension phase for eligible patients where as those subjects not enrolling in the extension study, a safety follow-up visit was conducted 20 weeks after the last dose of study medication [10]. The BE ABLE study included 250 patients with chronic plaque psoriasis with an affected body surface area of at least $10 \%$ and PASI of at least 12 . Patients were randomized 1:1:1:1:1:1 to receive subcutaneous bimekizumab every 4 weeks at doses of $64 \mathrm{mg}, 160 \mathrm{mg}, 160 \mathrm{mg}$ with $320 \mathrm{mg}$ loading dose, $320 \mathrm{mg}, 480$ $\mathrm{mg}$, or placebo, subcutaneously [10]. Primary endpoint of this study was the achievement of PASI90 response at week 12 . The reduction of at least 2 categories or the achievement of clear or almost clear skin condition as defined by Investigator's Global Assessment (IGA) at week 8 and at week 12, PASI90 response at week 8, PASI75 response at week 12, and PASI100 response at week 12, represented the secondary endpoints [10]. Seventy-nine percent and $60 \%$ of patients obtained PASI90 and PASI100 responses at week 12, respectively. Magnitude as well as rapidity of clinical response was impressive as demonstrated by the high number of PASI90 responders at week 8 (up to 86\%), PASI75 (up to 93\%) and PASI100 (up to 60\%) at week 12 [10]. Clinical efficacy was significantly higher compared to placebo in terms of PASI and IGA reduction [10]. Safety data set showed treatment-emergent adverse events (TEAEs) in $61 \%$ of bimekizumab-treated patients vs. $36 \%$ of placebo-treated patients. The most commonly reported TEAEs $(>5 \%$ patients in any group) at week 12 were represented by nasopharyngitis, upper respiratory tract infections, arthralgia, $\gamma$-glutamyltransferase increase, respiratory tract infection, neutropenia, rhinitis, tonsillitis, hypertension, oral candidiasis, headache, leukopenia, and vomiting [10]. Fungal infections including oral candidiasis, oral fungal infection, vulvovaginal mycotic infection, and tinea pedis were reported in 9 (4.3\%) bimekizumab-treated patients and they could be expected because of the protective role of both IL-17 cytokines against fungal infections. AEs led to treatment discontinuation in $4.8 \%$ of bimekizumab-treated patients and in $2.4 \%$ of the placebo group [10].

${ }^{\star}$ Correspondence to: Dermatology Unit, Department of Clinical and Experimental Medicine, University of Pisa, Ospedale Santa Chiara, Edificio 11, Via Roma 67, 56126 Pisa, Italy, E-mail: m.romanelli@med.unipi.it

Key words: psoriasis; psoriatic arthritis; bimekizumab; bispecific agent

Received: August 02, 2018; Accepted: August 17, 2018; Published: August 20, 2018 
Clinical studies testing bimekizumab for the treatment of psoriatic arthritis: A phase Ib, double-blind, placebo-controlled, proofof-concept trial randomized PsA patients to bimekizumab $(\mathrm{n}=39)$ or placebo $(n=14)$ for 20 weeks [11]. Different bimekizumab dose regimens were tested concomitantly with the use of anti-inflammatory and antirheumatic products, folic acid, analgesics, and DMARDs. Disease severity was assessed by the American College of Rheumatology (ACR) score. Patients receiving bimekizumab had greater joint improvement obtaining higher rates of ACR20 (at least 20\% improvement of baseline ACR score) response, ACR50, and ACR70, compared with placebo [11]. Primary efficacy endpoint (ACR20 at week 8) was obtained in $80 \%$ of patients, ACR50 in 40\%, and ACR70 in 23\% (ACR70) [11]. ACR50 and ACR70 response rates were more frequently observed at week12 and week 16 , in $57 \%$ and $37 \%$ of patients, respectively [11]. Notably, clinical response was evident as early as week 2 .

In patients with skin involvement $\geq 3 \%$ body surface area, week 8 response rates for PASI75 and PASI 100 were $100 \%$ and $87 \%$, respectively [11]. No unexpected safety signals were detected, with the majority of TEAEs classified as mild or moderate, while serious AEs were not related to the study drug [11]. A more recently performed multi-center, randomized, double-blind, placebo-controlled, parallel-group, doseranging, phase IIb (BE ACTIVE) study enrolling 206 patients with PsA assigned to bimekizumab or placebo during 12-week double-blind treatment period, followed by re-randomization to the different doseblind bimekizumab treatment groups for 36 weeks [12]. The overall treatment duration was 48 weeks. ACR50 (primary endpoint) response was obtained in up to $46 \%$ of bimekizumab-treated patients, compared to $7 \%$ of placebo-treated patients, at week 12 [12]. These results were achieved in a mixed patient population constituted by both biologic naïve and biologic-exposed patients [12].

Bimekizumab-treated patients with skin involvement $\geq 3 \%$ body surface area, in up to $60 \%$ of cases experienced a complete skin clearance (PASI100), while up to 65\% had PASI90 response (secondary endpoint) vs. $7 \%$ of the placebo group, at week $12[12,13]$.

\section{Conclusion}

Other bispecific biologic agents have been developed for the treatment of psoriasis and they are now being tested in clinical trials [14]. Nevertheless, bimekizumab is the first bispecific biologic agent entering phase III program. These preliminary data remarkably demonstrated an elevated and rapid efficacy of bimekizumab in clearing skin and improving joint inflammation. It would be of interest to have confirmatory data from the 5 trials constituting the current phase III program, in particular from the head-to-head studies comparing bimekizumab with ustekinumab (NCT03370133), adalimumab (NCT03412747), or secukinumab (NCT03536884) [15-17].

\section{References}

1. Gladman DD, Antoni C, Mease P, Clegg DO, Nash P (2005) Psoriatic arthritis: epidemiology, clinical features, course, and outcome. Ann Rheum Dis 64: 14-17. [Crossref]

2. Chiricozzi A, Romanelli P, Volpe E, Borsellino G, Romanelli M (2018) Scanning the Immunopathogenesis of Psoriasis. Int J Mol Sci 19. [Crossref]

3. Monin L, Gaffen SL (2018) Interleukin 17 Family Cytokines: Signaling Mechanisms, Biological Activities, and Therapeutic Implications. Cold Spring Harb Perspect Biol 10: $\mathrm{a} 028522$.

4. Wright JF, Bennett F, Li B, Brooks J, Luxenberg DP, et al. (2008) The human IL17F/IL-17A heterodimeric cytokine signals through the IL-17RA/IL-17RC receptor complex. J Immunol 181: 2799-2805. [Crossref]

5. Maroof A, Smallie T, Archer S, Simpson CL, Griffiths M, et al. (2017) Dual IL-17A and IL-17F inhibition with bimekizumab provides evidence for IL-17F contribution to immune-mediated inflammatory skin response. J Invest Dermatol 137 : S120.

6. Maroof A, Baeten D, Archer S, Griffiths M, Shaw S (2017) IL-17F contributes to human chronic inflammation in synovial tissue: preclinical evidence with dual IL-17A and IL-17F inhibition with bimekizumab in psoriatic arthritis. Ann Rheum Dis 76 : A13-A.

7. Maroof A, Okoye R, Smallie T, Baeten D, Archer S, et al. (2017) Bimekizumab dual inhibition of IL-17A and IL-17F provides evidence of IL-17F contribution to chronic inflammation in disease-relevant cells. Ann Rheum Dis $76: 213$.

8. van Baarsen LG, Lebre MC, van der Coelen D, Aarrass S, Tang MW, et al. (2014) Heterogeneous expression pattern of interleukin 17A (IL-17A), IL-17F and their receptors in synovium of rheumatoid arthritis, psoriatic arthritis and osteoarthritis: possible explanation for nonresponse to anti-IL-17 therapy? Arthritis Res Ther 16 : 426. [Crossref]

9. Glatt S, Helmer E, Haier B, Strimenopoulou F, Price G, et al. (2017) First-in-human randomized study of bimekizumab, a humanized monoclonal antibody and selective dual inhibitor of IL-17A and IL-17F, in mild psoriasis. Br J Clin Pharmacol 83 : 9911001. [Crossref]

10. Papp KA, Merola JF, Gottlieb AB, Griffiths CEM, Cross N, et al. (2018) Dual neutralization of both interleukin 17A and interleukin $17 \mathrm{~F}$ with bimekizumab in patients with psoriasis: Results from BE ABLE 1, a 12-week randomized, doubleblinded, placebo-controlled phase 2b trial. J Am Acad Dermatol 79 : 277-286. [Crossref]

11. Glatt S, Baeten D, Baker T, Griffiths M, Ionescu L, et al. (2018) Dual IL-17A and IL-17F neutralisation by bimekizumab in psoriatic arthritis: evidence from preclinical experiments and a randomised placebo-controlled clinical trial that IL-17F contributes to human chronic tissue inflammation. Ann Rheum Dis $77: 523-532$. [Crossref]

12. https://www.ucb.com/stories-media/Press-Releases/article/Bimekizumab...sive-jointand-skin-responses-for-psoriatic-arthritis-patients-nbsp

13. https://www.mdedge.com/edermatologynews/article/158562/psoriatic-arthritis/herecomes-bimekizumab-newest-il-17-inhibitor

14. Torres T, Romanelli M, Chiricozzi A (2016) A revolutionary therapeutic approach for psoriasis: bispecific biological agents. Expert Opin Investig Drugs 25: 751-4. [Crossref]

15. https://clinicaltrials.gov/ct2/show/NCT03370133?term=bimekizumab\&phase $=2 \&$ rank $=5$

16. https://clinicaltrials.gov/ct2/show/NCT03412747?term=bimekizumab\&phase=2\&rank=4

17. https://clinicaltrials.gov/ct2/show/NCT03536884?term=bimekizumab\&phase=2\&rank=3

Copyright: (2018 Chiricozzi A. This is an open-access article distributed under the terms of the Creative Commons Attribution License, which permits unrestricted use, distribution, and reproduction in any medium, provided the original author and source are credited. 